\title{
NOTE ON THE TAYLOR EXPANSION OF SMOOTH FUNCTIONS DEFINED ON SOBOLEV SPACES
}

\author{
By \\ Atsushi YoshIKAwA*
}

\section{§1. Introduction}

It is well-known that the Sobolev spaces $H^{\sigma}\left(\boldsymbol{R}^{n}\right)$ (with norm $\|\cdot\|_{\sigma}$ ) are multiplicative algebras when $\sigma>n / 2$. Let $u \in H^{\sigma}\left(\boldsymbol{R}^{n}\right)$ be real valued. If $f$ is a rapidly decreasing function on the real line, i.e., $f \in \mathcal{S}(\boldsymbol{R})$, then we may speak of the composite function $f(u)$, which again belongs to $H^{\sigma}\left(\boldsymbol{R}^{n}\right)$ provided $f(0)=0$ (See Rauch and Reed [1]). As for more precise results including higher order Taylor expansions, we have the following

Theorem. Suppose $\sigma>(n / 2)+1$, and $u$ and $v \in H^{\sigma}\left(\boldsymbol{R}^{n}\right)$ are real valued. Let $f \in \mathcal{S}(\boldsymbol{R})$. Consider the $m$-th remainder

$$
R_{m}(f)(v ; u)=f(v+u)-\sum_{k=0}^{m-1} \frac{1}{k !} f^{(k)}(v) u^{k}
$$

of the Taylor expansion of $f(v+u)$ around $u=0(m=1,2, \cdots)$. Then $R_{m}(f)(v ; u)$ $\in H^{\sigma}\left(\boldsymbol{R}^{n}\right)$ and, for $0 \leqq s \leqq \sigma$,

$$
\begin{gathered}
\left\|R_{m}(f)(v ; u)\right\|_{s} \leqq A_{m, s}\left(1+\|v\|_{\operatorname{Max}(s, \sigma-1)}+\|v\|_{0}\|\nabla v\|_{\sigma-1}^{\operatorname{Max}_{0}(s, \sigma-1)}\right) \\
\times\left(\frac{1}{m !}\left\|u^{m}\right\|_{s}+\frac{1}{(m+1) !}\|u\|_{(2 m)}^{m}\|\nabla u\|_{\sigma-1}^{\operatorname{Max}(s, 1)}\right),
\end{gathered}
$$

where $A_{m, 8}$ is a positive constant independent of $u$ and $v$. In the above, $\nabla$ stands for the gradient operator, and $\|w\|_{(p)}=\left(\int_{R}|w(x)|^{p} d x\right)^{1 / p}$ is the $L^{p}$-norm of a function $w$ on $\boldsymbol{R}^{n}, p>0$. Note $\|w\|_{(2)}=\|w\|_{0}$, for $H^{0}\left(\boldsymbol{R}^{n}\right)=L^{2}\left(\boldsymbol{R}^{n}\right)$.

REMARKS. (i) $\|u\|_{(2 m)}$ makes sense for $u \in H^{\sigma}\left(\boldsymbol{R}^{n}\right)$ since $\sigma>(n / 2)+1$ and $H^{\sigma}\left(\boldsymbol{R}^{n}\right) \subset H^{n(m-1) / 2 m}\left(\boldsymbol{R}^{n}\right) \subset L^{2 m}\left(\boldsymbol{R}^{n}\right)$ by the Sobolev embedding theorem.

(ii) The constant $A_{m, s}$ admits the estimate

$$
A_{m, s} \leqq C_{s} \frac{1}{2 \pi} \int_{R}|\hat{f}(\tau)||\tau|^{m}\left(1+|\tau|^{s *}\right) d \tau,
$$

\footnotetext{
* Supported in part by Grant-in-Aid for Scientific Research, Ministry of Education, Science and Culture, Japanese Government, No. 62460005.

Received February 8, 1990. Revised June 1, 1990.
} 
$s^{*}=1+\operatorname{Max}(s, 1)+\operatorname{Max}(s, \sigma-1)$. Here $\hat{f}(\tau)$ is the Fourier transform of $f$ and $C_{s}$ a positive constant independent of $m$ and of $f$.

(iii) Similar results are valid when $\sigma>n / 2$ and $\sigma \geqq 1$. Then we have to replace (1.2) by

$$
\begin{aligned}
& \left\|R_{m}(f)(v ; u)\right\|_{s} \leqq A_{m, s, \varepsilon}\left(1+\|v\|_{\sigma}+\|v\|_{0}\|\nabla v\|_{\sigma-1}^{\sigma / \varepsilon}\right) \\
& \quad \times\left(\frac{1}{m !}\left\|u^{m}\right\|_{s}+\frac{1}{(m+1) !}\|u\|_{(2 m)}^{m}\|\nabla u\|_{\sigma-1}^{\max (s / \varepsilon, 1)}\right),
\end{aligned}
$$

where

$$
A_{m, s, \varepsilon} \leqq C_{s, \varepsilon} \frac{1}{2 \pi} \int_{R}|\hat{f}(\tau)||\tau|^{m}\left(1+|\tau|^{s *(\varepsilon)}\right) d \tau,
$$

$s^{*}(\varepsilon)=1+(\sigma / \varepsilon)+\operatorname{Max}(s / \varepsilon, 1), 0<\varepsilon<\sigma-(n / 2), \varepsilon \leqq 1$.

The proof of Theorem is carried out by extending the idea of Rauch and Reed [1] where they discussed the case of $m=1$ and $v=0, f(0)=0$. Observe

$$
R_{m}(f)(v ; u)=\frac{1}{2 \pi} \int_{R} e^{i v \tau}\left(e^{i u \tau}-\sum_{k=0}^{m-1} \frac{(i \tau u)^{k}}{k !}\right) \hat{f}(\tau) d \tau,
$$

where $\hat{f}(\tau)=\int_{R} e^{-i \tau t} f(t) d t$ is the Fourier transform of $f(t)$. Then, for $0 \leqq s \leqq \sigma$

$$
\left\|R_{m}(f)(v ; u)\right\|_{s}=\frac{1}{2 \pi} \int_{R}\left\|e^{i v \tau}\left(e^{i u \tau}-\sum_{k=0}^{m-1} \frac{(i \tau u)^{k}}{k !}\right)\right\|_{s}|\hat{f}(\tau)| d \tau .
$$

Therefore, in order to prove Theorem, we only have to verify the estimate:

$$
\begin{aligned}
& \left\|e^{i v \tau}\left(e^{i u \tau}-\sum_{k=0}^{m-1} \frac{(i \tau u)^{k}}{k !}\right)\right\|_{s} \\
& \quad \leqq C_{s}\left(1+\|v\|_{\operatorname{Max}(s, \sigma-1)}+\|v\|_{0}\|\nabla v\|_{\sigma-1}^{\operatorname{Max}(s, \sigma \quad 1)}\right) \\
& \quad \times\left(\frac{1}{m !}\left\|u^{m}\right\|_{s}+\frac{1}{(m+1) !}\|u\|_{(2 m)}^{m}\|\nabla u\|_{\sigma-1}^{\operatorname{Max}(s, 1)}\right)\left(1+|\tau|^{s *}\right)|\tau|^{m},
\end{aligned}
$$

for real $\tau$ provided $u, v \in H^{\sigma}\left(\boldsymbol{R}^{n}\right), \sigma>(n / 2)+1$, are real valued. Here $s^{*}=$ $1+\operatorname{Max}(s, 1)+\operatorname{Max}(s, \sigma-1)$ and $C_{s}$ is a positive constant independent of $u, v, \tau$ and $m$.

For a verification of (1.4), we appeal to the following

LEMma 1.1. Suppose $\sigma>(n / 2)+1$, and $m$ a positive integer. Let $w \in H^{\sigma}\left(\boldsymbol{R}^{n}\right)$ be real valued. Then $e^{i v}-\sum_{k=0}^{m-1}(i w)^{k} / k ! \in H^{\sigma}\left(\boldsymbol{R}^{n}\right)$ and

$$
\begin{aligned}
& \left\|e^{i w}-\sum_{k=0}^{m-1} \frac{(i w)^{k}}{k !}\right\|_{s} \\
& \quad \leqq C_{s}\left(\frac{1}{m !}\left\|w^{m}\right\|_{s}+\frac{1}{(m+1) !}\|w\|_{(2 m)}^{m}\|\nabla w\|_{\sigma-1}^{\operatorname{Max}(s, 1)}\right),
\end{aligned}
$$


for $0 \leqq s \leqq \sigma$. Here $C_{s}$ is a positive constant independent of $m$ and $w$.

A proof will be given in the next section.

Let us derive (1.4) for $\tau=1$ from (1.5), since then (1.4) for general $\tau$ follows via an elementary inequality:

$$
\left(1+r^{a} X+r^{a+b} Y\right)\left(r^{d} Z+r^{c+d} W\right) \leqq r^{d}\left(1+r^{a+b+c}\right)(1+X+Y)(Z+W),
$$

for all $r>0$. Here $a, b, c, d, X, Y, Z, W$ are all positive. Observe the identity:

$$
\begin{aligned}
& e^{i v}\left(e^{i u}-\sum_{k=0}^{m-1} \frac{(i u)^{k}}{k !}\right) \\
& \quad=\left(e^{i v}-1\right)\left(e^{i u}-\sum_{k=0}^{m-1} \frac{(i u)^{k}}{k !}\right)+\left(e^{i u}-\sum_{k=0}^{m-1} \frac{(i u)^{k}}{k !}\right) .
\end{aligned}
$$

In view of Lemma 1.1, we only need to show

$$
\left\|\left(e^{i v}-1\right) w\right\|_{s} \leqq C_{s}\left(\|v\|_{\operatorname{Max}(s, \sigma-1)}+\|v\|_{0}\|\nabla v\|_{\sigma-1}^{\operatorname{Max}(s, \sigma-1)}\right)\|w\|_{s}
$$

for all $w \in H^{s}\left(\boldsymbol{R}^{n}\right), 0 \leqq s \leqq \sigma$, when $v$ is real valued. Now by Lemma 1.1 and the Sobolev embedding theorem,

$$
\left\|\left(e^{i v}-1\right) w\right\|_{0} \leqq C\left\|e^{i v}-1\right\|_{\sigma-1}\|w\|_{0} \leqq C\left(\|v\|_{\sigma-1}+\|v\|_{0}\|\nabla v\|_{\sigma-1}^{\sigma-1}\right)\|w\|_{0},
$$

while, for $\sigma \geqq s \geqq \sigma-1$,

$$
\left\|\left(e^{i v}-1\right) w\right\|_{s} \leqq C\left\|e^{i v-}-1\right\|_{s}\|w\|_{s} \leqq C\left(\|v\|_{s}+\|v\|_{0}\|\nabla v\|_{\sigma-1}^{s}\right)\|w\|_{s} .
$$

(1.6) then follows by interpolating $0 \leqq s \leqq \sigma-1$.

REMARK. We also have $\left\|\left(e^{i v}-1\right) w\right\|_{0} \leqq 2\|w\|_{0}$ since $v$ is real valued. Thus, when $\|v\|_{\sigma-1}+\|v\|_{0}\|\nabla v\|_{\sigma-1}^{\sigma-1}$ is very large, we have

$$
\left\|\left(e^{i v}-1\right) w\right\|_{s} \leqq C\left(\|v\|_{\sigma-1}+\|v\|_{0}\|\nabla v\|_{\sigma-1}^{\sigma-1}\right)^{s /(\sigma-1)}\|w\|_{s},
$$

for $0 \leqq s \leqq \sigma-1$.

\section{§ 2. Proof of Lemma 1.1}

Our proof of Lemma 1.1 is based on the following simplified analogue of Proposition 4.1 of Rauch and Reed [1].

LEMmA 2.1. Suppose $g \in H^{\sigma}\left(\boldsymbol{R}^{n}\right)$ is real valued. Let $0 \leqq s \leqq \sigma$. Then

$$
\left|\operatorname{Re}\left(i\langle D\rangle^{s} M_{g}\langle D\rangle^{-s} w, w\right)\right| \leqq B_{s}\|\nabla g\|_{\sigma_{-1}}\|w\|_{-1}\|w\|_{0},
$$

for all $w \in H^{0}\left(\boldsymbol{R}^{n}\right)$ provided $\sigma>(n / 2)+1$. Here $B_{s}$ is a positive constant independent of $w$ and $g$ and (,) the inner product of $H^{0}\left(\boldsymbol{R}^{n}\right)$. Recall $M_{g}$ is the multi- 
plication operator by the function $g$, and $\langle D\rangle^{s}$ is the pseudo-differential oterator with the full symbol $\langle\xi\rangle^{s}=\left(1+|\xi|^{2}\right)^{s / 2}, \xi \in \boldsymbol{R}^{n}$.

PROOF. Since $g$ is real valued,

$$
\operatorname{Re} i\left(\langle D\rangle^{s} M_{\boldsymbol{g}}\langle D\rangle^{-s} w, w\right)=\operatorname{Re} i\left(\left[\langle D\rangle^{s}, M_{\boldsymbol{g}}\right]\langle D\rangle^{-s} w, w\right) \text {. }
$$

Then (2.1) is shown by the classical estimate (See, e.g., [2]):

$$
\begin{aligned}
& \left|\left(v,\left[\langle D\rangle^{s}, M_{g}\right] u\right)\right| \leqq C\|\nabla g\|_{\sigma-1}\left(\|v\|_{0}\|u\|_{s^{\prime}}+\|v\|_{-t^{\prime}}\|u\|_{s}\right), \\
& s^{\prime} \geqq s-1, \quad 1 \geqq t^{\prime} \geqq 0, \quad \sigma-\frac{n}{2}>t^{\prime}, \quad \sigma-\frac{n}{2}>s-s^{\prime}, \quad \sigma \geqq 1, \quad s>0 .
\end{aligned}
$$

We can choose $s^{\prime}=s-1, t^{\prime}=1$ if $\sigma>(n / 2)+1$. If we merely have $\sigma>n / 2$, $\sigma \geqq 1$, then we choose $s^{\prime}=s-\varepsilon, t^{\prime}=\varepsilon$ for $\sigma-(n / 2)>\varepsilon>0,1 \geqq \varepsilon>0$.

Now let us proceed to a verification of Lemma 1.1. The case when $m=1$ is essentially due to Rauch and Reed [1]. By slightly modifying their ideas, a proof of Lemma 1.1 for general $m$ is obtained. Thus, to verify (1.5), we first reproduce a part of the discussions of Rauch and Reed [1], and then indicate our modification. Let

$$
E_{m}(w)=e^{i w}-\sum_{k=0}^{m-1} \frac{(i w)^{k}}{k !}, \quad m=1,2, \cdots,
$$

and

$$
W_{m}(t)=\langle D\rangle^{s} E_{m}(t w) .
$$

A straightforward computation yields to

$$
\frac{d}{d t} W_{m}(t)=i\langle D\rangle^{s} M_{w}\langle D\rangle^{-s} W_{m}(t)+\frac{t^{m-1}}{(m-1) !}\langle D\rangle^{s}(i w)^{m},
$$

with $W_{m}(0)=0$. Taking the inner product of the both hand sides with $W_{m}(t)$, and using Lemma 2.1 we have,

$$
\frac{d}{d t}\left\|W_{m}(t)\right\|_{0} \leqq B_{s}\|\nabla w\|_{\sigma-1}\left\|W_{m}(t)\right\|_{-1}+\frac{t^{m-1}}{(m-1) !}\left\|w^{m}\right\|_{s} .
$$

Our idea is to employ the logarithmic convexity of the Sobolev scale. Thus, suppose $s>1$. Then

$$
\left\|W_{m}(t)\right\|_{-1}=\left\|E_{m}(t w)\right\|_{s-1} \leqq\left\|E_{m}(t w)\right\|_{0}^{1-\theta}\left\|E_{m}(t w)\right\|_{s}^{\theta},
$$

$\theta=1-1 / s$. Therefore, for any $\delta>0$,

$$
\left\|W_{m}(t)\right\|_{-1} \leqq \delta^{\theta} \frac{t^{m}}{m !}\|w\|_{(2 m)}^{m}+C_{\theta} \delta^{\theta-1}\left\|W_{m}^{\prime}(t)\right\|_{0} .
$$

Here we have used the fact $\left\|E_{m}(t w)\right\|_{0} \leqq\left(t^{m} / m !\right)\|w\|_{(2 m)}^{m}$, which is also a con- 
sequence of realness of $w$. It follows

$$
\begin{aligned}
\frac{d}{d t}\left\|W_{m}(t)\right\|_{0} \leqq & C_{\theta} B_{s}\|\nabla w\|_{\sigma-1} \delta^{\theta-1}\left\|W_{m}(t)\right\|_{0} \\
& +B_{s} \delta^{\theta}\|\nabla w\|_{\sigma-1} \frac{t^{m}}{m !}\|w\|_{(2 m)}^{m}+\frac{t^{m-1}}{(m-1) !}\left\|w^{m}\right\|_{s} .
\end{aligned}
$$

Since Lemma 1.1 is trivial when $w=0$, we assume $w \neq 0$ so that $\nabla w \neq 0$. Choose $\delta=\|\nabla w\|_{\sigma-1}^{\boldsymbol{s}}$. Then

$$
\begin{aligned}
\frac{d}{d t}\left\|W_{m}(t)\right\|_{0} \leqq & C_{\theta} B_{s}\left\|W_{m}(t)\right\|_{0} \\
& +B_{s}\|\nabla w\|_{\sigma-1}^{s}\|w\|_{(2 m)}^{m} \frac{t^{m}}{m !}+\left\|w^{m}\right\|_{s} \frac{t^{m-1}}{(m-1) !}
\end{aligned}
$$

Therefore, integrating from $t=0$ to $t=1$, we have

$$
\left\|E_{m}(w)\right\|_{s}=\left\|W_{m}(1)\right\|_{0} \leqq B_{s} e^{C_{\theta} B_{s}} \frac{1}{(m+1) !}\|w\|_{(2 m)}^{m}\|\nabla w\|_{\sigma-1}^{s}+e^{C_{\theta} B_{s}} \frac{1}{m !}\left\|w^{m}\right\|_{s} .
$$

On the other hand, if $s \leqq 1$, then

$$
\left\|W_{m}(t)\right\|_{-1}=\left\|\langle D\rangle^{s-1} E_{m}(t w)\right\|_{0} \leqq\left\|E_{m}(t w)\right\|_{0} \leqq \frac{t^{m}}{m !}\|w\|_{(2 m)}^{m} .
$$

Thus, (2.2) yields to

$$
\frac{d}{d t}\left\|W_{m}(t)\right\|_{0} \leqq B_{s} \frac{t^{m}}{m !}\|\nabla w\|_{\sigma-1}\|w\|_{(2 m)}^{m}+\frac{t^{m-1}}{(m-1) !}\left\|w^{m}\right\|_{s},
$$

whence

$$
\left\|E_{m}(w)\right\|_{s} \leqq \frac{B_{s}}{(m+1) !}\|\nabla w\|_{\sigma-1}\|w\|_{(2 m)}^{m}+\frac{1}{m !}\left\|w^{m}\right\|_{s}
$$

\section{References}

[1] J. Reed and M. Rauch, Nonlinear microlocal analysis of semilinear hyperbolic system in one space dimension, Duke Math. J., 49 (1982), 337-475.

[2] A. Yoshikawa, On expansions of commutators acting in the Sobolev scale (preprint). 